\title{
Scanning tunneling spectroscopy and Dirac point resonances due to a single Co adatom on gated graphene
}

\author{
Alireza Saffarzadeh ${ }^{1,2}$ and George Kirczenow ${ }^{2}$ \\ ${ }^{1}$ Department of Physics, Payame Noor University, P.O. Box 19395-3697 Tehran, Iran \\ ${ }^{2}$ Department of Physics, Simon Fraser University, Burnaby, British Columbia, Canada V5A $1 S 6$
}

(Dated: Monday $13^{\text {th }}$ August, 2018)

\begin{abstract}
Based on the standard tight-binding model of the graphene $\pi$-band electronic structure, the extended Hückel model for the adsorbate and graphene carbon atoms, and spin splittings estimated from density functional theory (DFT), the Dirac point resonances due to a single cobalt atom on graphene are studied. The relaxed geometry of the magnetic adsorbate and the graphene is calculated using DFT. The system shows strong spin polarization in the vicinity of the graphene Dirac point energy for all values of the gate voltage, due to the spin-splitting of Co $3 d$ orbitals. We also model the differential conductance spectra for this system that have been measured in the scanning tunneling microscopy (STM) experiments of Brar et al. [Nat. Phys. 7, 43 (2011)]. We interpret the experimentally observed behavior of the S-peak in the STM differential conductance spectrum as evidence of tunneling between the STM tip and a cobalt-induced Dirac point resonant state of the graphene, via a Co $3 d$ orbital. The cobalt ionization state which is determined by the energy position of the resonance can be tuned by gate voltage, similar to that seen in the experiment.
\end{abstract}

\section{INTRODUCTION}

Graphene, a single atomic layer of graphite, has attracted a great amount of attention because of its fundamental interest and promising applications [1-4]. One of the remarkable properties of pristine graphene is the linear dispersion relation of its electronic spectrum near the Dirac point $[5,6]$. It has been demonstrated theoretically that chemical adatoms, whether magnetic [7-16] or nonmagnetic [16-29], adsorbed on graphene can induce resonant states in the vicinity of the Dirac point energy, and these states may give rise to strong scattering of electrons in the graphene. However, to our knowledge, there has as yet been no direct experimental observation of these Dirac point resonances. The presence of chemical adsorbates, atomic or molecular, on graphene strongly affects electronic properties and makes graphene-based devices suitable for chemical sensing [29-36].

Furthermore, the chemical potential of graphene electrons can be easily tuned by a gate voltage, and thus, the adsorbate ionization state can be externally controlled $[12,13]$. Using single and few-layer graphene films on top of a $\mathrm{SiO}_{2}$ substrate, it has been demonstrated that a graphene field-effect transistor can switch between two dimensional electron and hole gases by changing the gate voltage [37]. It has been shown that, using an electrochemically top-gated graphene transistor, even much higher electron and hole doping can be induced than by standard $\mathrm{SiO}_{2}$ back-gating [38]. On the other hand, an insulating state with large suppression of conductivity can be created in bilayer graphene field-effect transistors by using a top gate in addition to the global back gate [39]. When an electric field was applied perpendicular to the bilayer, a gap of less than $10 \mathrm{meV}$ at low temperatures [39] and up to $0.25 \mathrm{eV}$ at room temperature [40] opened in the bilayer graphene, the size of the gap being tunable by the electric field.
On the other hand, according to density functional theory (DFT) calculations, cobalt adatoms on graphene show the highest magnetic anisotropy energy relative to other $3 d$ transition metals adsorbed on graphene [41, 42]. Thus, the Co-graphene system has potential applications in magnetic data storage. Recently, scanning tunneling microscopy (STM) and spectroscopy measurements demonstrated that individual Co atoms deposited onto back-gated graphene devices can be controllably ionized by application of a gate voltage or STM tip bias voltage [43]. Several different features were observed in the gate-dependence of the measured differential conductance. Inelastic electron tunneling and hybrid electronicvibrational states were suggested as possible explanations for some of these features. Therefore, the effects of inelastic scattering of electrons should be included in any theoretical study whose objective is to reproduce these features of the calculated inelastic tunneling spectra [4447]. A different feature is related to a sharp peak in the spectrum which moves in the opposite direction in energy than the graphene Dirac point energy in response to changes in the gate voltage.

In a previous theoretical study, using DFT electronic structure calculations in a generalized gradient approximation for periodic systems, Jacob and Kotliar [12] showed that a single Co atom most likely adsorbs at the hollow site of a clean graphene sheet. They found that the coupling of the Co $3 d$ levels to the graphene substrate and the dynamic correlations are strongly dependent on the orbital symmetry, temperature, distance of the Co atom from the graphene sheet, and gate voltage. More recently, Chan et al. [13] investigated the gated Co-graphene system in a supercell structure with periodic boundary conditions using the local density approximation $(\mathrm{LDA})+U$ method, with several values of the $U$ parameter. The projected density of states was calculated for different $U$ values and back gating treated qualitatively by $\mathrm{LDA}+U$ calculations for different inte- 
ger charge states of the sample per unit cell. An ionization effect for the Co adatom was found as the charge state of the whole system was varied. No calculated STM current-voltage characteristics were reported, so the calculated local density of states (LDOS) could not be compared with STM data. Hence, the true LDOS of the Cographene system remains uncertain. Note that experimental and theoretical studies of the interaction between Co and graphene have shown charge-transfer to occur between the graphene and $\mathrm{Co}$, resulting in the graphene being electron doped [48-50].

The aim of this paper is to reproduce some resonance features seen in the STM experiment by means of DFT calculations of the relaxed geometry and the extended Hückel model of quantum chemistry [51-53] for the electronic structure of Co adatom on graphene with DFTbased spin splitting parameters. To this end, we study resonant scattering of spin-up and spin-down electrons by the single Co atom adsorbed on gated graphene for electron energies close to the Dirac point. The electronic density of states for the Co-graphene system is calculated and the dependence of the resonant states on the gate voltage is discussed. In addition, we investigate the differential conductance of electron tunneling through the Co adatom as a function of sample bias and gate voltage which has not been addressed in the previous theories. Our transport calculations show that the experimentally observed behavior of the previously unidentified S-peak in the STM differential conductance spectrum [43] is consistent with this feature being due to tunneling between the STM tip and a cobalt-induced Dirac point resonant state of the graphene, via a Co $3 d$ orbital. In this study, we do not consider the Kondo effect of Co on graphene $[9,10,12]$, because this is a many-body effect and outside of the scope of the present study.

The paper is organized as follows. In Sec. II, we present the model Hamiltonian of the infinite 2D graphene sheet with a single cobalt adatom. We show that the tight-binding Hamiltonian for the system can be transformed into an effective graphene Hamiltonian that includes the effect of the Co adatom. In Sec. III, we derive an exact relation for the graphene Green's function matrix elements in the presence of the adatom using $T$-matrix theory. The results for the spin- and gate-dependent $T$-matrix and the electronic density of states will be presented in Sec. IV, where the adsorbateinduced resonance states are discussed. The effect of local rehybridization of the graphene from the $s p^{2}$ to $s p^{3}$ electronic structure on the Dirac point resonances, that occurs when the Co atom bonds covalently to the graphene, is also examined. In Sec. V, we present a formalism suitable for studies of the gate-induced ionization of a single Co adatom on graphene, seen in the experiment. We calculate the gate dependence of the differential conductance by means of the Landauer-Büttiker formalism based on the Green's function technique, and the extended Hückel theory and DFT-based spin splitting parameters and compare the results with the STM measurements of Brar et al.[43]. Finally, in Sec. VI we conclude this work with a general discussion of the results.

\section{THE MODEL}

Our calculations are performed using ab initio geometry relaxation based on density functional theory for a Co adsorbate on the honeycomb graphene lattice using the Gaussian 09 software package together with the HSEh1PBE hybrid function and the 6-311G(d) Gaussian basis set [51-53]. Such a relaxed geometry is expected to be accurate because the density functional theory on which this relaxation is based, has already been well optimized for carrying out accurate ground state total energy calculations. We found that the lowest-energy binding site is the $\mathrm{H}$-site in which the adsorbate is positioned at height $h_{0}=1.68 \AA$ above the middle of a hexagon of the graphene, as shown in Fig. 1. The reason is that, the $\mathrm{H}$-site has the largest number of neighboring $\mathrm{C}$ atoms, lowering the adsorption energy. The structure studied was a graphene disk consisting of 54 carbon atoms passivated at the edges with 18 hydrogen atoms and the Co atom being bonded to the graphene at the center of the disk. All the atoms were allowed to relax freely. Although a finite size cluster of carbon atoms was used in the calculations, the bond lengths between the Co atom and its neighboring $\mathrm{C}$ atoms were well converged which indicates that $54 \mathrm{C}$ atoms is large enough to model the graphene plane. The final relaxed structure obtained in this way is shown in Fig. 1. From the figure, it is clear that, there is no distortion out of plane of the graphene structure due to the Co adsorbate. This is in agreement with the previous studies of Co-graphene systems in periodic supercell structures $[7,13]$. In addition, we found in-plane distortion of $0.01 \AA$ in bond length between carbon atoms of central hexagon relative to their nearest neighbor carbon atoms in the graphene plane. The existence of an in-plane distortion equal to $0.023 \AA$ in the periodic Co-graphene supercell has also been reported by Liu et al. [16].

We describe the present system by a tight-binding model Hamiltonian derived from extended Hückel theory in a basis of extended molecular orbitals (EMOs) which are linear combinations of the atomic valence orbitals of the adatom and the $2 \mathrm{~s}, 2 p_{x}$, and $2 p_{y}$ orbitals of the graphene carbon atoms. We refer the reader to Ref. [29] for a detailed explanation regarding tight-binding models for adsorbates on graphene derived from extended Hückel theory and to Refs. 54-56 for further discussions of the application of extended Hückel theory to graphene-based systems and carbon nanotubes. Accordingly, the EMOs together with the $2 p_{z}$ orbitals of the graphene carbon atoms form the basis set for our total Hamiltonian of the Co-graphene system that can be written in the form

$$
H=H_{0}+\sum_{\alpha \sigma} \epsilon_{\alpha \sigma} d_{\alpha \sigma}^{\dagger} d_{\alpha \sigma}+\sum_{\alpha, \sigma, n} \gamma_{\alpha n, \sigma}\left(d_{\alpha \sigma}^{\dagger} a_{n \sigma}+\text { h.c. }\right)
$$




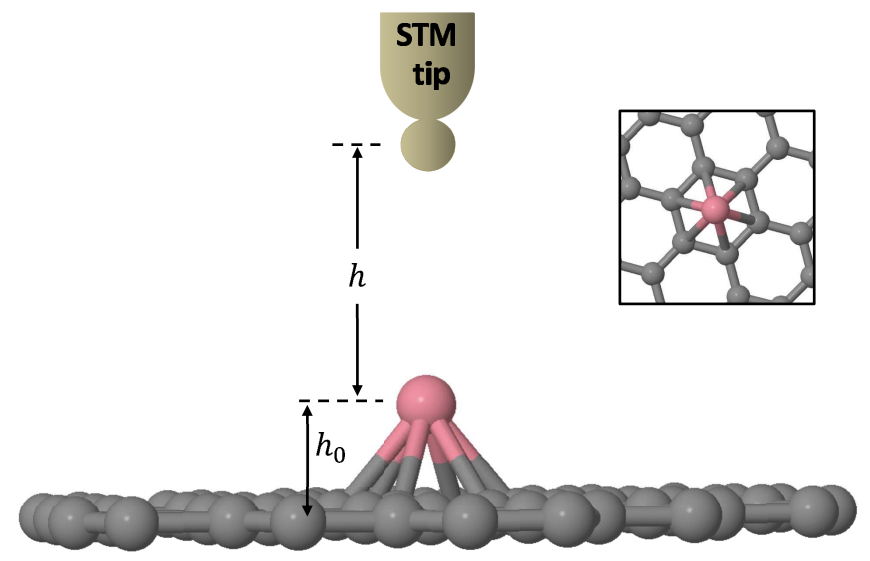

FIG. 1. (Color online) Side view of the relaxed geometry for a single adsorbed Co atom on graphene. The optimal height $h_{0}=1.68 \AA$ is the perpendicular distance between the Co adsorbate and graphene plane, while $h=4 \AA$ is the distance between the adsorbate and STM tip which is directly above the Co atom. The inset shows the top view of the Co-graphene system. The hydrogen atoms at the edges of graphene are not shown here.

where the first term corresponds to the Hamiltonian of clean graphene in the basis of $2 p_{z}$ carbon orbitals, $a_{n \sigma}$ is the annihilation operator for an electron with spin $\sigma$ in the $2 p_{z}$ orbital $\phi_{n \sigma}$ of carbon atom $n, d_{\alpha \sigma}^{\dagger}$ is the creation operator for an electron in an EMO $\psi_{\alpha \sigma}$ of the Co adatom and $\epsilon_{\alpha \sigma}$ is the corresponding energy eigenvalue. $\gamma_{\alpha n, \sigma}$ is the spin-dependent matrix element of the extended Hückel Hamiltonian between the $2 p_{z}$ orbital of carbon atom $n$ and the $\operatorname{EMO} \alpha$. We note that, since the basis set used in the extended Hückel theory is nonorthogonal, it is possible for the overlap $s_{\alpha n, \sigma}=\left\langle\psi_{\alpha \sigma} \mid \phi_{n \sigma}\right\rangle$ between the $2 p_{z}$ orbital $\phi_{n \sigma}$ of carbon atom $n$ and EMO $\psi_{\alpha \sigma}$ to be non-zero. Because of this, we replace $\gamma_{\alpha n, \sigma}$ in the Hamiltonian by $\gamma_{\alpha n, \sigma}-\epsilon s_{\alpha n, \sigma}$ where $\epsilon$ is the electron energy $[57,58]$. Henceforth we will omit the spin index $\sigma$ in the overlap parameter and show it as $s_{\alpha n}$ for simplicity.

The unperturbed Hamiltonian of two dimensional graphene, $H_{0}$, which can be modeled on a honeycomb lattice of two nonequivalent sites (that is, $A$ and $B$ ) per unit cell is expressed as [6]

$$
H_{0}=-t \sum_{\langle i, j\rangle}\left[a_{i}^{\dagger} a_{j}+\text { h.c. }\right]
$$

and describes the motion of carriers between $2 p_{z}$ carbon orbitals of graphene with a nearest-neighbor hopping parameter $t$. We neglect the small changes in $t$ due to the above mentioned in-plane distortion in the graphene geometry, induced by the Co atom. We note that although Eq. (2) does not include the overlaps between the nearest neighbor $2 p_{z}$ orbitals of the carbon atoms explicitly, it is a widely used form of the Hamiltonian that has been optimized to provide an accurate description of the graphene $\pi$-band states around the Dirac point.[6] We adopt it in the present work for that reason. Furthermore, since it is assumed that in clean graphene the spectrum is spindegenerate, for simplicity we have suppressed the spin indices in Eq. (2). Here $a_{i}^{\dagger}$ is the creation operator for an electron in $2 p_{z}$ carbon orbital $i$. The Hamiltonian for graphene is diagonal when written in the basis $|\mathbf{k}, c(v)\rangle$ defined as $|\mathbf{k}, c(v)\rangle=\left(|\mathbf{k}, A\rangle \pm e^{-i \phi(\mathbf{k})}|\mathbf{k}, B\rangle\right) / \sqrt{2}$ where $A$ and $B$ stand for $A$ and $B$ sublattices and

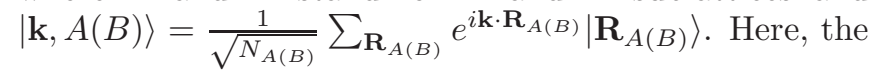
$c(v)$ sign denotes the conduction (valence) band, $\left|\mathbf{R}_{A(B)}\right\rangle$ represents an atomic orbital centered at a lattice site $A(B)$ and $N_{A(B)}$ is the total number of $A(B)$-lattice points. The phase $\phi(\mathbf{k})$ is the polar angle for momentum $\mathbf{k}$ and is defined as $\phi(\mathbf{k})=\operatorname{Im}(\ln f(\mathbf{k}))$ where $f(\mathbf{k})=e^{i k_{x} a / \sqrt{3}}+2 e^{-i k_{x} a / 2 \sqrt{3}} \cos \left(k_{y} a / 2\right)$. The corresponding eigenvalues in terms of $f(\mathbf{k})$ can be expressed as $\varepsilon_{c(v)}= \pm t \sqrt{|f(\mathbf{k})|^{2}}[5,6]$.

The carbon $2 s, 2 p_{x}$, and $2 p_{y}$ orbitals in graphene combine to form in-plane $\sigma$ (bonding) and $\sigma^{*}$ (anti-bonding) orbitals. In this study the $2 s, 2 p_{x}$, and $2 p_{y}$ orbitals of the graphene were shifted down in energy relative to their energies in extended Hückel model so as to yield an energy gap between the graphene $\sigma$ and $\pi$ bands in agreement with the results of the $a b$ initio band structure calculations for graphene [62].

The electron eigenfunctions of the total Hamiltonian can be written in terms of $2 p_{z}$ carbon orbitals and the adsorbed atom EMOs. In this regard, it has been shown $[21,29]$ that there is a relationship between $2 p_{z}$ orbitals of graphene and the adsorbate orbitals, so that, the total Hamiltonian Eq. (1) can be replaced by an effective Hamiltonian which consists of the graphene $2 p_{z}$ Hamiltonian with an energy-dependent potential on each carbon atom to which the adsorbed atom binds. In fact, the energy-dependent effective potential acts like a self-energy that an adsorbate induces on electrons in graphene. For a single Co adatom that bonds to six neighboring graphene carbon atoms, the effective Hamiltonian can be written as [29]

$$
\begin{gathered}
H_{\mathrm{eff}}=H_{0}+V \\
V=\sum_{n, m=1}^{6} \sum_{\sigma} V_{n m, \sigma} a_{n \sigma}^{\dagger} a_{m \sigma}
\end{gathered}
$$

where $a_{n \sigma}$ is the annihilation operator for an electron in the $2 p_{z}$ orbital of carbon atom $n$ and $V_{n m, \sigma}=$ $\sum_{\alpha} \gamma_{\alpha n, \sigma} \gamma_{\alpha m, \sigma}^{*} /\left(\epsilon-\epsilon_{\alpha \sigma}\right)$ displays a resonant energy dependence. Here, the summation is over the extended molecular orbitals $\alpha$ of the Co adatom that bonds to carbon atoms $m$ and $n$ of the graphene. In this study, we consider 171 EMOs for the adsorbed Co atom on graphene which are linear combinations of the Co $3 d$, $4 s$ and $4 p$ valence orbitals and the $2 \mathrm{~s}, 2 p_{x}, 2 p_{y}$ valence orbitals of 54 carbon atoms shown in Fig. 1. The spin dependence of $\epsilon_{\alpha \sigma}$ and $\gamma_{\alpha n, \sigma}$ originates from the spin splitting of the Co $4 s$ and $3 d$ orbitals. Due to the crystal field 
splitting, the Co $3 d$ orbitals split into three symmetry groups: the singly degenerate $A_{1}$ group which only consists of $3 d_{3 z^{2}-r^{2}}$ orbital, a doubly degenerate $E_{1}$ group consisting of the $3 d_{z x}$ and $3 d_{z y}$ orbitals, and a doubly degenerate $E_{2}$ group derived from the $3 d_{x y}$ and $3 d_{x^{2}-y^{2}}$ orbitals. The spin splitting values appear as extra energies that modify the Co $4 s$ and $3 d$ orbitals on-site energies obtained from the extended Hückel theory parameters. The value of these splittings are calculated from each of two major peaks in spin density of states spectrum for Co from Ref. [13]. In addition, since in the parameters obtained from the extended Hückel theory the effect of all carbon atoms (neighbors) is included in the calculations, the Dirac point of graphene will shift somewhat $(0.072 t)$ from zero energy to higher energy. To compensate for this effect, the cobalt energy levels are also shifted in energy by the same amount.

\section{T-MATRIX AND UNPERTURBED GREEN'S FUNCTION}

To study the strength of scattering associated with a single Co adatom on graphene we use the $T$-matrix formalism which is defined as $G^{\text {eff }}=G^{0}+G^{0} T G^{0}$ where $G^{\mathrm{eff}}=\left(\epsilon+i \eta-H_{\mathrm{eff}}\right)^{-1}$ is the effective Green's function for the graphene in the presence of the Co adatom, and $G^{0}=\left(\epsilon+i \eta-H_{0}\right)^{-1}$ is the unperturbed Green's function (i.e. graphene Green's function in the absence of Co adsorbate) for $\pi$-band electrons. The $T$-matrix is related to $G^{0}$ and $V$ by $T=V\left(1-G^{0} V\right)^{-1}$ where all the operators are $6 \times 6$ matrices with the basis of the graphene $p_{z}$ orbitals of the carbon atoms to which the Co atom binds. To obtain the $T$-matrix components we need the matrix elements of $G_{0}$. Since, $\varepsilon_{v}(\mathbf{k})=-\varepsilon_{c}(\mathbf{k})$ the matrix elements of $\left\langle i\left|G^{0}(\epsilon)\right| j\right\rangle=G_{i j}^{0}(\epsilon)$ between two arbitrary sites $i$ and $j$ of a graphene sheet can be written as

$$
G_{i j}^{0}(\epsilon)=\sum_{\mathbf{k}}\left[\frac{\langle i \mid \mathbf{k},+\rangle\langle\mathbf{k},+\mid j\rangle}{\epsilon+i \eta-\varepsilon_{c}(\mathbf{k})}+\frac{\langle i \mid \mathbf{k},-\rangle\langle\mathbf{k},-\mid j\rangle}{\epsilon+i \eta+\varepsilon_{c}(\mathbf{k})}\right]
$$

where the sum is over the Brillouin zone, $\epsilon$ is the energy and $\eta$ is a positive infinitesimal. The results for $G_{i j}^{0}(\epsilon)$ depend on whether the sites $i$ and $j$ correspond to two equivalent or inequivalent sites. In the case of equivalent sites, the diagonal and off-diagonal matrix elements can be obtained from the following equation

$$
G_{i j}^{0}(\epsilon)=\frac{1}{N} \sum_{\mathbf{k}}\left[\frac{\epsilon}{(\epsilon+i \eta)^{2}-\varepsilon_{c}^{2}(\mathbf{k})}\right] e^{i \mathbf{k} \cdot\left(\mathbf{R}_{i}-\mathbf{R}_{j}\right)}
$$

where $N=N_{A(B)}$. For the case of inequivalent sites, the off-diagonal matrix elements of $G^{0}$ corresponding to two inequivalent atoms ( $A$ and $B$ in the same cells or in different cells) are written as:

$$
G_{i j}^{0}(\epsilon)=\frac{1}{N} \sum_{\mathbf{k}}\left[\frac{-\varepsilon_{c}(\mathbf{k})}{(\epsilon+i \eta)^{2}-\varepsilon_{c}^{2}(\mathbf{k})}\right] e^{i\left[\mathbf{k} \cdot\left(\mathbf{R}_{i}-\mathbf{R}_{j}\right)+\phi(\mathbf{k})\right]}
$$

We note that for the cases of adsorbates such as $\mathrm{H}$ and $\mathrm{OH}$ that bond to only one carbon atom and also for $\mathrm{O}$ adatom which bonds to two neighboring carbon atoms, by expanding the band structure of graphene close to the Dirac points (at the K and K' points in the Brillouin zone), one can linearize the dispersion relation and obtain analytic expressions for the required matrix elements that is valid in the energy range $|\epsilon| / t \leq 0.8$ (see Ref. [29]). For a general matrix element of the Green's function, however, there is no such analytic expression, and we therefore consider the full $\pi$-band structure of graphene and evaluate numerically the above Green's function matrix elements which are valid in all energy ranges.

The locations of resonance energies are determined by the poles of $T$-matrix, which can be different for the spinup and spin-down states. By taking matrix elements of the effective Green's function between the graphene $2 p_{z}$ orbitals of the six carbon atoms to which the Co atom binds, we obtain

$$
G_{i j, \sigma}^{\mathrm{eff}}(\epsilon)=G_{i j}^{0}(\epsilon)+\sum_{l l^{\prime}=1}^{6} G_{i l}^{0}(\epsilon) T_{l l^{\prime}, \sigma}(\epsilon) G_{l^{\prime} j}^{0}(\epsilon)
$$

where $T_{l l^{\prime}, \sigma}(\epsilon)$ are the spin- and energy-dependent $T$ matrix elements which describe resonant scattering of electrons in graphene due to the adsorbate levels renormalized by hybridization with $2 p_{z}$ orbital of carbon atoms. The effect of this hybridization usually appears as a shift in the resonance levels towards the Dirac point of graphene [21, 29].

\section{SPIN-DEPENDENT DIRAC POINT RESONANCE OF CO ADATOM}

\section{A. T-matrix feature}

To investigate the scattering effects associated with a single Co adatom on graphene, we study the square modulus of the $T$-matrix. The scattering will be a spindependent process due to the spin-splitting of Co $4 s$ and $3 d$ orbitals, and the scattering at the energies $\epsilon$ at which $\left|T_{\sigma}(\epsilon)\right|^{2}$ has maxima is of the resonant type. The energies of these resonant states can be found by setting the denominator of the square modulus of the $T$-matrix equal to zero, i.e. $\left|\operatorname{det}\left(1-G^{0} V\right)\right|^{2}=0$. Based on the $\gamma_{\alpha n, \sigma}$ and $\epsilon_{\alpha n, \sigma}$ parameters obtained from the present model, the square modulus of the $T$-matrix for spin-up and spindown electrons is shown vs. the electron energy $\epsilon$ in Fig. 2. The two studied models (solid and dashed curves) are explained in detail below. Note that, the effect of overlaps $s_{\alpha n}$ between the EMOs $\psi_{\alpha}$ and the $2 p_{z}$ orbitals $\phi_{n}$ of carbon atoms $n$ are included in all of the calculations.

The solid curves correspond to a model in which all 171 EMOs are used in the calculations. In other words, the EMOs are linear combinations of the $4 s, 4 p_{x}, 4 p_{y}$, $4 p_{z}, 3 d_{x^{2}-y^{2}}, 3 d_{x y}, 3 d_{3 z^{2}-r^{2}}, 3 d_{z x}, 3 d_{z y}$ atomic valence 


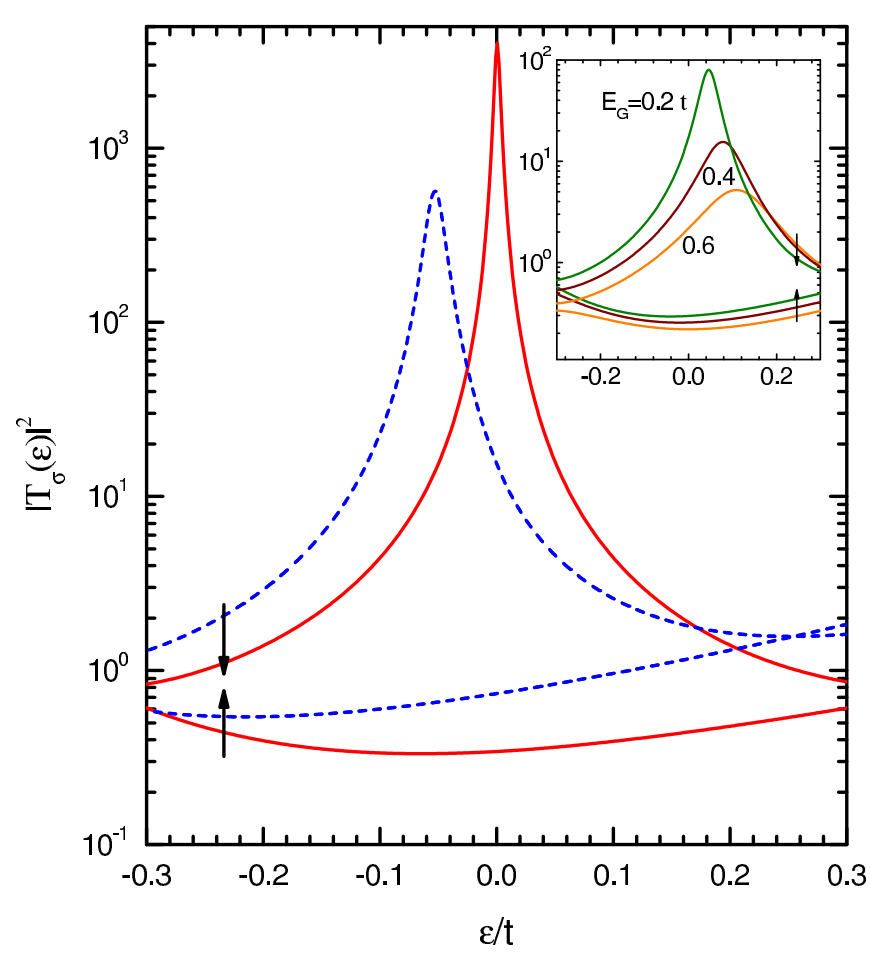

FIG. 2. (Color online) Spin-dependence of square modulus of $T$-matrix vs. electron energy at the gate voltage for which $E_{G}$, the gate induced shift in energy of the graphene orbitals relative to those of the $\mathrm{Co}$, is zero. Solid lines correspond to a case in which the EMOs are linear combinations of the atomic valence orbitals of the adsorbed Co atom and the $2 s$, $2 p_{x}$, and $2 p_{y}$ valence orbitals of each of the $\mathrm{C}$ atoms, while the dashed lines are related to a case in which only the valence orbitals of the adsorbate (no carbon orbitals) are included in the EMOs. The symbol $\uparrow(\downarrow)$ corresponds to spin-up (spindown) electrons. The inset shows the effect of gate voltage on the square modulus of $T$-matrix when the $2 s, 2 p_{x}$, and $2 p_{y}$ orbitals of carbon atoms are included in the calculations. $T_{\sigma}$, $\epsilon$ and $E_{G}$ are in units of $t=2.7 \mathrm{eV}$.

orbitals of the adsorbed Co atom and the $2 s, 2 p_{x}$, and $2 p_{y}$ valence orbitals of all 54 carbon atoms. The square modulus of the $T$-matrix of the spin-down electrons displays a resonant peak at the Dirac point of graphene, while for the spin-up electrons there is no such a resonance feature in the shown energy interval $(|\epsilon| / t \leq 0.3)$. This is a measure of high spin polarization in the system.

The dashed curves correspond to a simpler model of the EMOs in which no carbon orbitals are taken into account in the EMO calculation and only 9 orbitals due to the Co atom are included in the EMOs. We see that, the omission of the graphene carbon $2 s, 2 p_{x}$ and $2 p_{y}$ valence orbitals in the calculations and of the coupling of adsorbate to these orbitals (which are involved in the local rehybridization of the graphene from $s p^{2}$ to $s p^{3}$ bonding near the adsorbed Co atom) shifts the position of the adsorbate-induced Dirac point resonance energy to $\epsilon_{D R}=-0.053 t$ and its intensity is about one order of magnitude weaker than that found in the more complete model.

In earlier work $[25,29,60,61]$ it has been shown that the adsorption of $\mathrm{H}, \mathrm{F}, \mathrm{OH}$, and $\mathrm{O}$ atoms on graphene distorts the graphene plane by shifting carbon atoms out of the plane. The omission of the graphene carbon $2 s$, $2 p_{x}$, and $2 p_{y}$ valence orbitals from EMOs was found to affect the adsorbate-induced resonances for these adsorbates very strongly [29]. On the other hand, we have already explained that there is no out of plane structural distortion due to the Co adsorption on graphene, while if these valence orbitals are omitted from the EMOs $\psi_{\alpha}$, the Co resonance state is still affected significantly. Since the inclusion of $2 s, 2 p_{x}$, and $2 p_{y}$ valence orbitals in the EMOs plays a dominant role for the Co adsorbate on graphene, and these orbitals play a crucial role in the rehybridization of graphene from $s p^{2}$ to $s p^{3}$ bonding, it seems reasonable to infer that significant rehybridization from the $s p^{2}$ to the $s p^{3}$ electronic structure occurs for this system, despite the absence of structural out of plane distortion of the graphene.

In the experiment of Brar et al. [43] both a back gate and the STM tip played the role of gates. Together they induced electrostatic potential differences between the graphene plane and the adsorbed Co atom that manifested themselves in the experimental STM data [43]. We model this gate-induced potential difference phenomenologically by shifting the carbon orbitals energies, derived from the extended Hückel theory, and also the electronic structure of graphene $\pi$-band used in the unperturbed Green's function of the graphene plane, $G^{0}$, by a gate voltage-dependent amount $E_{G}$ relative to the energies of the Co orbitals. Thus $E_{G}$ acts as a proxy for the gate voltage in the present work. Accordingly, $E_{G}=0$ corresponds to the gate voltage at which the graphene Dirac point and the resonant state due to the adsorbate are located at zero energy $\epsilon / t=0.0$. The gate voltage at which $E_{G}=0$ is an important reference point for the gate-controlled ionization of the Co atom, especially when we compare our differential conductance results with the STM and spectroscopy measurements in the Sec. V. We emphasize that, in our calculations, the extended Hückel model parameters describing both the on-site and intersite Hamiltonian matrix elements are recalculated for each value of the gate voltage. The dependence of the intersite Hamiltonian matrix elements on the gate voltage[59] implies that hybridization between the orbitals on different atoms depends on the gate voltage in the present theory. We note that previous work of Sofo et al. $[60,61]$ has indicated that doping can lead to a strong change in hybridization of the graphene.

The application of a gate voltage in the experiment [43] caused the Co density of states features to move relative to the Fermi energy and hence, the Co levels could be emptied or filled with electron carriers. [43] The movement of a prominent peak (marked S in the STM conductance spectra shown in Figs. 2a and $2 \mathrm{~b}$ in Ref. [43]) relative to the Dirac point with changing gate voltage can be interpreted as further evidence of a Co en- 
TABLE I. Minimal set of effective tight-binding parameters $\epsilon_{\alpha \sigma}, \gamma_{\alpha n, \sigma}$ and $s_{\alpha n}$ in units of $t=2.7 \mathrm{eV}$ for adsorbed Co atom on graphene at zero gate voltage. The spin-dependent EMO energies $\epsilon_{\alpha \sigma}$ are measured from the Dirac point energy of graphene.

\begin{tabular}{|c|c|c|c|c|c|c|c|c|c|c|c|c|}
\hline$\epsilon_{\alpha \uparrow}$ & $\gamma_{\alpha 1 \uparrow}$ & $\gamma_{\alpha 2 \uparrow}$ & $\gamma_{\alpha 3 \uparrow}$ & $\gamma_{\alpha 4 \uparrow}$ & $\gamma_{\alpha 5 \uparrow}$ & $\gamma_{\alpha 6 \uparrow}$ & $s_{\alpha 1 \uparrow}$ & $s_{\alpha 2 \uparrow}$ & $s_{\alpha 3 \uparrow}$ & $s_{\alpha 4 \uparrow}$ & $s_{\alpha 5 \uparrow}$ & $s_{\alpha 6 \uparrow}$ \\
\hline-0.855 & -0.084 & -0.078 & 0.162 & -0.078 & -0.079 & 0.157 & 0.023 & 0.022 & -0.044 & 0.022 & 0.022 & -0.043 \\
\hline-0.855 & 0.135 & .142 & -0.001 & & -0.135 & 0.003 & -0.037 & & & & & -0.001 \\
\hline-0.704 & 0.175 & 0.239 & 0.067 & -0.173 & -0.244 & -0.068 & 0.044 & -0.061 & -0.017 & & 0.062 & 0.017 \\
\hline-0.704 & 0.177 & -0.062 & -0.236 & -0.180 & 0.061 & 0.244 & -0.045 & & 0.060 & & -0.015 & -0.061 \\
\hline 1.363 & -0.296 & -0.293 & -0.292 & -0.296 & -0.298 & -0.300 & 0.102 & 0.1 & 0.101 & 0.102 & & 0.103 \\
\hline 2.513 & -0.043 & -0.276 & -0.246 & & 0.2 & & & & & & & -0.144 \\
\hline 2.513 & -0.290 & -0.117 & 0.171 & 0.295 & 0.125 & -0.172 & 0.1 & & -0 . & & & 0.108 \\
\hline 3.201 & 0.161 & 0.158 & 0.157 & 0.162 & 0.165 & 0.166 & -0.087 & -0.086 & -0.085 & -0.087 & -0.0 & -0.089 \\
\hline 4.291 & 0.39 & 0 & 0.3 & 0.3 & 0.4 & 0.4 & 0 & -0 . & -0 . & 00 & & -0.207 \\
\hline 8.174 & -0.144 & -0.161 & -0.162 & -0.148 & -0.130 & -0.129 & 0.071 & 0.074 & 0.074 & 0.072 & & 0.068 \\
\hline$\epsilon_{\alpha \downarrow}$ & $\gamma_{\alpha 1 \downarrow}$ & $\gamma_{\alpha 2 \downarrow}$ & $\gamma_{\alpha 3 \downarrow}$ & $\gamma_{\alpha 4 \downarrow}$ & $\gamma_{\alpha 5 \downarrow}$ & $\gamma_{\alpha 6 \downarrow}$ & & & & & & $s_{\alpha 6 \downarrow}$ \\
\hline-0.494 & 0.084 & 0.088 & -0.172 & 0.074 & 0.090 & -0.163 & -0.022 & -0.023 & 0.0 & -0.019 & -0.023 & 0.042 \\
\hline-0.494 & 0.144 & -0.148 & -0.009 & 0.151 & -0.137 & -0.003 & -0.038 & & 0.002 & -0.039 & 36 & 0.001 \\
\hline-0.121 & 0.128 & 0.263 & 0.140 & -0.121 & -0.271 & -0.144 & -0.029 & -0. & & & & 33 \\
\hline-0.121 & -0.234 & -0.008 & 0.220 & 0.238 & 0.013 & -0.232 & & & -0 . & -0 . & -0 . & 0.053 \\
\hline 1.518 & 0.263 & 0.262 & 0.261 & 0.264 & 0.265 & 0.266 & -0.092 & -0.092 & -0.092 & -0.0 & & -0.093 \\
\hline 2.514 & -0.042 & -0.278 & -0.248 & 0.017 & & & & & & -0.012 & -0.163 & -0.145 \\
\hline 2.514 & 0.293 & 0.118 & -0.172 & -0.297 & -0.126 & 0.173 & -0.182 & -0.074 & & & 0.078 & -0.108 \\
\hline 3.210 & 0.155 & 0.152 & 0.151 & 0.155 & 0.159 & 0.160 & -0.0 & -0.0 & -0.0 & -0.0 & -0. & -0.086 \\
\hline & & & 0.379 & & 0.406 & & & & & & & -0.212 \\
\hline 8.186 & 0.146 & 0.163 & 0.164 & 0.149 & 0.132 & 0.131 & -0.073 & -0.076 & -0.076 & -0.074 & -0.071 & -0.071 \\
\hline
\end{tabular}

ergy level moving relative to the graphene $\pi$ band as the gate voltage was varied. The strength of the conductance peak $\mathrm{S}$ was observed to increase greatly in the experiment (Fig. 2a in Ref. [43]) as the peak approached the graphene Dirac point (Fig. 2b in Ref. [43]). As will be discussed in Section V, we interpret this behavior as being due to a Co resonant level approaching the graphene Dirac point as $E_{G}$ approaches zero. As this happens the cobalt-induced resonance feature for spin-down graphene electrons (associated with the peak in the square modulus of $T$-matrix in Fig.2) also approaches at the Dirac point as $E_{G}$ strengthens. However with increasing $\left|E_{G}\right|$, the probability for overlap between the resonant cobalt level for spin-down electrons and the graphene states in the vicinity of the Dirac point decreases and the sharp conductance peak is suppressed in a similar way to the rather broad peak (marked S) in Fig. 2 in Ref. [43] for the higher absolute values of the gate voltage.

The inset of Fig. 2 shows the calculated $E_{G}$ dependence of the square modulus of the $T$-matrix and indicates that the electron energy $\epsilon_{D R}$ at which the resonance occurs and the strength of electron scattering depends on the value of gate voltage. We have mentioned above that a sharp resonance peak centered at $\epsilon_{D R}=0.0 t$ appears at the gate voltage for which $E_{G}=0$ due to spin-down electrons. However, with increasing $E_{G}$ the strength of resonance peak in the inset of Fig. 2 decreases and the peak position shifts towards the higher energies; $\epsilon_{D R}=0.045 t, 0.078 t, 0.11 t$ at $E_{G}=0.2 t, 0.4 t, 0.6 t$ respectively. In the case of decreasing $E_{G}$ the peak position $\epsilon_{D R}$, shifts to lower energies. For spin-up electrons the variation of the $\left|T_{\sigma}(\epsilon)\right|^{2}$ function vs. energy is approximately the same in the all gate voltages. The reason of such a behavior can be understood in terms of LDOS of the Co adsorbate and graphene sheet that will be explained below.

The EMO energies $\epsilon_{\alpha \sigma}$, hopping $\gamma_{\alpha n, \sigma}$ and overlap $s_{\alpha n}$ parameters for a minimal tight-binding model Hamiltonian that describes the Dirac point resonant state involved in the spin-dependent square modulus of the $T$ matrix and graphene LDOS at zero gate voltage are presented in Table I.

\section{B. Local Density of States}

The calculated spin-dependent LDOS for graphene in the presence of an adsorbed Co atom, and for the Co $4 s, A_{1}, E_{1}$, and $E_{2}$ orbitals at different gate voltages are shown in Fig. 3. For greater clarity, the electronic states within an energy window close to the Dirac point of graphene are also shown separately [see Fig. 3(a2)-(d2)]. In the case of the graphene LDOS, we used the same extended Hückel-theory-based model in the calculations that was used to calculate the square modulus of the $T$ matrix. Thus, the model includes the effects of the local rehybridization of the graphene plane due to the presence of the Co adatom. The LDOS in this case is defined as $D_{i, \sigma}(\epsilon)=-\operatorname{Im}\left[\left\langle i\left|G_{\sigma}^{\mathrm{eff}}(\epsilon)\right| i\right\rangle\right] / \pi$, where $G_{\sigma}^{\mathrm{eff}}(\epsilon)$ is given by Eq.(8) and $|i\rangle$ represents a $2 p_{z}$ orbital of a carbon atom. The solid curves in Fig. 3 correspond to the $2 p_{z}$ orbital of a graphene carbon atom to which the Co atom bonds. On the other hand, for the LDOS of the Co adsorbate, we have used the Hamiltonian $\widetilde{H}_{\sigma}=H_{\sigma}^{\mathrm{Co}}+\Sigma_{\sigma}^{\mathrm{C}}(\epsilon)$ for an electron with spin $\sigma(=\uparrow$ or $\downarrow)$ where $H_{\sigma}^{\text {Co }}$ is the 


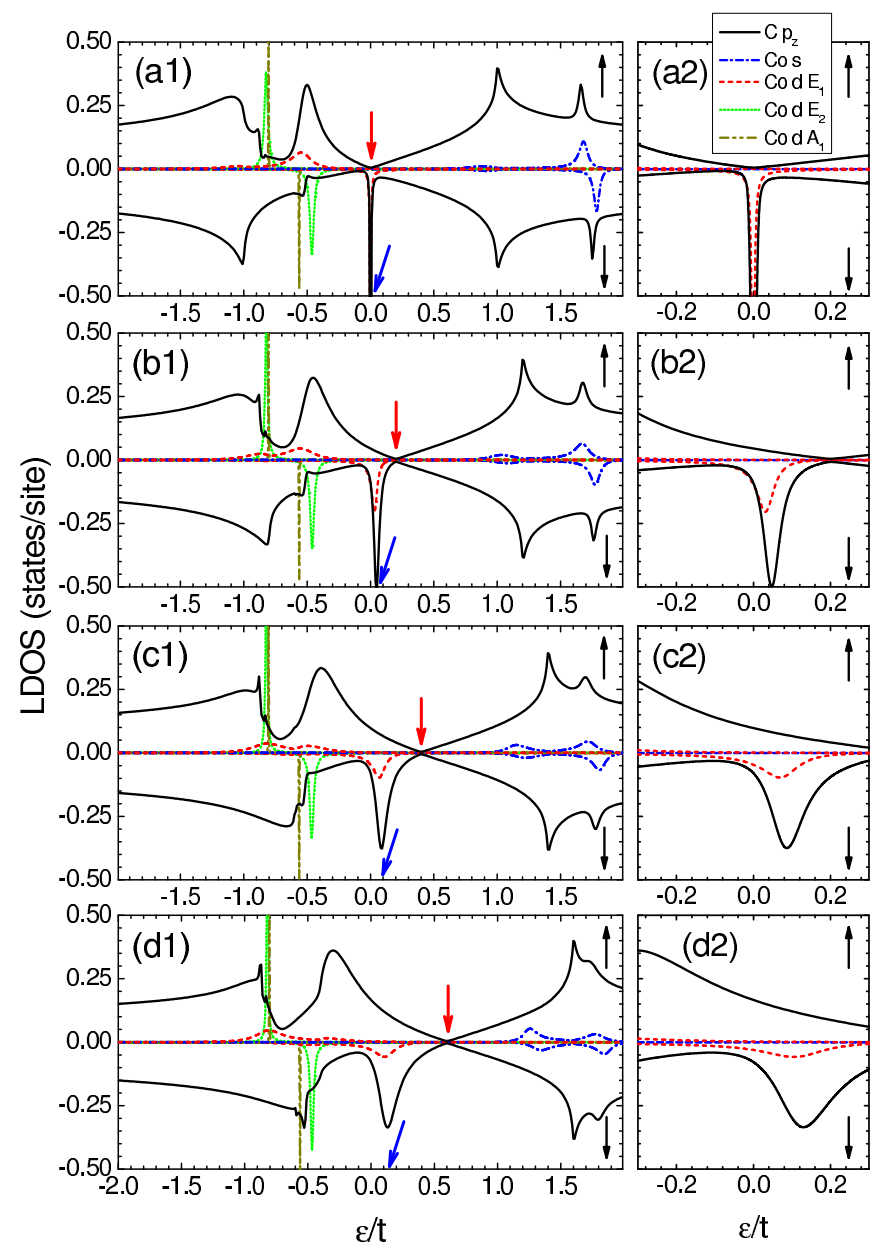

FIG. 3. (Color online) Calculated LDOS per site vs. electron energy $\epsilon$ for a single Co atom adsorbed on graphene at various gate voltages: (a1), (a2) $E_{G}=0.0 t$; (b1), (b2) $E_{G}=0.2 t,(\mathrm{c} 1),(\mathrm{c} 2) E_{G}=0.4 t ;(\mathrm{d} 1),(\mathrm{d} 2) E_{G}=0.6 t$. The symbol $\uparrow(\downarrow)$ corresponds to spin-up (spin-down) electrons. The LDOS associated with the $2 p_{z}$ orbital of a graphene carbon atom to which the Co atom bonds (solid black), Co $4 \mathrm{~s}$ (dash-dotted blue), Co $3 d A_{1}$ (dash-dot-dotted dark yellow), Co $3 d E_{1}$ (dashed red), and Co $3 d E_{2}$ (dotted green) states are shown. In Co LDOS, we multiplied by the scale factor $1 / 50$. The EMOs included in graphene LDOS calculation are linear combinations of the atomic valence orbitals of the Co atom and the $2 s, 2 p_{x}$, and $2 p_{y}$ valence orbitals of $\mathrm{C}$ atoms. For Co LDOS the effect of carbon $2 p_{z}$ orbitals is included as a selfenergy in the calculations. The red and blue arrows indicate the position of the graphene Dirac point and the graphene resonant state, respectively.

Hamiltonian of a free Co atom whose matrix elements are given by the extended Hückel theory, and the self-energy $\Sigma_{\sigma}^{\mathrm{C}}(\epsilon)=\tau_{\mathrm{Co}-\mathrm{C}, \sigma} G^{0}(\epsilon) \tau_{\mathrm{Co}-\mathrm{C}, \sigma}^{\dagger}$ contains the coupling effects of the adsorbate to the graphene carbon atoms [63]. Here, $\tau_{\mathrm{Co}-\mathrm{C}, \sigma}$ is a hopping matrix between the cobalt atom and the $2 p_{z}$ orbitals of the six carbon atoms of the graphene whose matrix elements are also given by the extended Hückel model parameters and $G^{0}(\epsilon)$ is the
Green's function of the clean graphene which is given by Eqs. (6) and (7). In the above self-energy, the effect of $2 s, 2 p_{x}$, and $2 p_{y}$ valence orbitals of $\mathrm{C}$ atoms could also be considered in the calculation. In such a case, however, the $\pi$-band Green's function, $G^{0}(\epsilon)$, is no longer sufficient and the graphene $\sigma$-band Green's function is required. Since the present self-energy is still able to reproduce the expected resonance features, we consider just the hopping parameters between the cobalt orbitals and the graphene $2 p_{z}$ orbitals in the Co LDOS calculation. Note that the overlaps $s_{\mathrm{Co}-\mathrm{C}}$ between the Co atom and the $2 p_{z}$ orbitals of the carbon atoms to which the adsorbate bonds are included in the calculations. Accordingly, the LDOS for the adsorbate is determined by $\widetilde{D}_{\ell, \sigma}(\epsilon)=-\operatorname{Im}\left[\left\langle\ell\left|\widetilde{G}_{\sigma}(\epsilon)\right| \ell\right\rangle\right] / \pi$, where $|\ell\rangle$ represents the $4 s, A_{1}, E_{1}$, or $E_{2}$ orbital, and $\widetilde{G}_{\sigma}(\epsilon)=\left(\epsilon+i \eta-\widetilde{H}_{\sigma}\right)^{-1}$ is the Green's function of the Co atom adsorbed on the graphene plane.

From Fig. 3 it is clear that the spin-down graphene states show a resonance at the energies close to the Dirac point that is due to coupling between the graphene and the Co $E_{1}$ orbital. This resonance is exactly positioned at the Dirac point energy at the gate voltage for which $E_{G}$, the gate induced shift in energy of the graphene orbitals relative to those of the Co, is zero. For this gate voltage the spin-down Co $E_{1}$ state coincides with the resonant graphene state that appears due to the presence of the Co adatom. Furthermore, because of the spin-splitting of the Co $3 d$ orbitals, the peak energy of the spin-up Co $E_{1}$ state is somewhat far (i.e., at approximately $-0.55 t$ for $\left.E_{G}=0\right)$ from the Dirac point energy and accordingly, only a relatively broad peak due to the spin-up Co $E_{1}$ state appears in the spectrum. The Co $A_{1}$ and $E_{2}$ orbitals are completely occupied for both spin states at the gate voltage for which $E_{G}=0$. Note that, the Fermi energy $E_{F}$ is set at the Dirac point of graphene, i.e. $E_{F}=0.0 t$. In this study, only the effect of positive $E_{G}$ is investigated. The reason for this will be explained in the next section. In both the graphene and Co LDOSs there is an energy shift with changing gate voltage. Although, as we mentioned above, the energy level shift due to the gate voltage is only applied on the graphene plane, the Co orbitals are also affected by this effect because, due to the non-zero overlap between the Co and graphene atomic orbitals, the effective hopping energies between the carbon and the Co orbitals depend on the on-site energies of the carbon orbitals $[57,58]$. In the energy window $(|\epsilon| / t \leq 0.3)$ that is shown in Fig. 3, the gate voltage decreases the occupation of both spin-up and spin-down graphene states by shifting the electronic states above the Fermi level $E_{F}$, and hence the occupation of spin-down $E_{1}$ orbital is also decreased. This result can be clearly seen in Fig. 3 where $E_{G}$ increases from $0.0 t$ to $0.2 t$ to $0.4 t$ and $0.6 t$ and the Dirac point of the graphene (indicated by red arrows) moves from zero energy to the values of $0.2 t, 0.4 t$ and $0.6 t$, respectively. Therefore, the Co orbitals which are coupled to the graphene shift somewhat to the higher energies and 
the Co $E_{1}$ orbital is gradually emptied of electrons. This result for Co $3 d$ orbitals is in agreement with the recent DFT calculations for gated Co atom on graphene in supercells with periodic boundary conditions [12, 13, 15]. Furthermore, by comparing Figs. 3(a)-3(d) it is clear that, with increasing $E_{G}$, the amplitudes of the Co $E_{1}$ and graphene $p_{z}$ density of states peaks decrease, the energy difference between the Dirac point and the graphene resonance state position increases and both the Co $E_{1}$ level and the graphene resonance state induced by $\mathrm{Co}$ adatom are broadened.

This indicates that the Co $E_{1}$ orbital is coupled strongly to the $\pi$-orbitals of graphene and a gate voltage can ionize the Co adatom through this orbital. This is in agreement with the work of Liu et al. [16] in which they reported that the hybridization between $E_{1}$ orbital of the $3 d$-transition-metal adatoms on graphene and $p_{z}$ orbitals of the graphene carbon atoms is strong and responsible for strong covalent bonding with graphene. By contrast, both the $A_{1}$ and $E_{2}$ orbitals are strongly localized and far from the Dirac point, however, the $A_{1}$ orbital hybridizes more weakly than the $E_{2}$ orbital with the graphene states. Note that, comparison of the work of Liu et al. [16] with the present results shows somewhat different coupling strengths of the Co $E_{2}$ orbital with $2 p_{z}$ orbitals of the carbon atoms which may be attributed to the periodic nature of the structures $(4 \times 4$ Co-graphene supercell) studied in their calculations. It may also be relevant that density functional theory-based electronic structure calculations are known to have fundamental limitations that can strongly affect the results that they yield for the coupling between adsorbed atoms and graphene; see Section VII of Ref. 29 for a discussion and specific examples. In addition, the spin-up and spin-down Co $4 s$ orbitals are far from the $E_{F}$; thus the occupation of these orbitals is zero at low gate voltages, although these orbitals hybridize strongly with the graphene states. This result is different than the results for periodic structures $[7,9,13]$ in which the Co $4 s$ orbital is close to the Dirac point energy and the effect of gating can ionize the Co atom by occupying or unoccupying the $4 s$ orbitals. In addition, in the periodic structures, the Dirac point resonances are broadened due to the presence of multiple adsorbed atoms on the graphene [7, 9, 13]. Therefore, it is evident that to better understand the features of adsorbate-induced Dirac point resonances and to verify the veracity of the theoretical predictions, comprehensive experimental investigations would be very important.

\section{SIMULATION OF STM MEASUREMENTS}

\section{A. Model}

In this section, assuming coherent transport, we describe how the electronic differential conductance $(d I / d V)$ through a Co atom adsorbed on a graphene plane can be calculated using the tight-binding Hamiltonians described in the preceding sections and the Landauer-Büttiker formalism [63]. The aim of this is to make a comparison with the STM differential conductance measurements in which the STM tip was held directly above the individual Co adatoms deposited onto a back-gated graphene device at $4.2 \mathrm{~K}$ [43]. To simulate the experimental condition, we model the STM tip (shown in Fig. 1) by a one-dimensional chain of Pt atoms with nearest neighbor hopping integral $t$. The Fermi energy is fixed at the center of the Pt chain band structure. For Pt atoms we use $6 s, 6 p_{x}, 6 p_{y}, 6 p_{z}, 5 d_{x^{2}-y^{2}}, 5 d_{x y}$, $5 d_{3 z^{2}-r^{2}}, 5 d_{z x}, 5 d_{z y}$ atomic valence orbitals. Since each $\mathrm{Pt}$ atom in the tip is described by 9 valence orbitals, then we model the tip by nine semi-infinite leads of orthogonal atomic orbitals, one orbital per lead site, with spacing (periodicity) $a$ between orbital sites. Each of the one-dimensional leads is decoupled from the others, thus, there is no hopping between different lead orbitals. According to the above description, the Green's function of the Co atom coupled to the graphene carbon atoms and the STM system can be written as

$$
G_{\sigma}(\epsilon, V)=\left[\epsilon+i \eta-H_{\sigma}^{\mathrm{Co}}-\Sigma_{\sigma}^{\mathrm{C}}(\epsilon)-\Sigma_{\sigma}^{\mathrm{STM}}(\epsilon+e V)\right]^{-1},
$$

where $\Sigma_{\sigma}^{\mathrm{STM}}(\epsilon)=\tau_{\mathrm{Co}-\mathrm{STM}, \sigma} g_{\mathrm{STM}}(\epsilon) \tau_{\mathrm{Co}-\mathrm{STM}, \sigma}^{\dagger}$ is a selfenergy for an electron with spin $\sigma$ and describes the coupling of the adsorbate to the STM tip, $\tau_{\mathrm{Co}-\mathrm{STM}, \sigma}$ is the hopping matrix between STM and the Co adatom whose matrix elements are given by the extended Hückel theory parameters, and $g_{\mathrm{STM}}=-(1 / t) e^{i k a} \mathbb{I}$ is the surface Green's function matrix for the semi-infinite leads where $k$ is the wave number in the leads and $\mathbb{I}$ is a $9 \times 9$ unit matrix $[63,64]$. The overlaps $s_{\mathrm{Co}-\mathrm{STM}}$ between the Co and $\mathrm{Pt}$ orbitals are included in the calculations. Now, if we make use of the nonequilibrium Green's function technique to obtain the spin current $I_{\sigma}$ for a sample bias $V$ between graphene and the tip [63, 64], then the spindependent conductance $d I_{\sigma} / d V$ for an electron with spin $\sigma$ is given by

$$
\mathcal{G}_{\sigma}(V) \simeq \frac{e}{h} \int_{-\infty}^{\infty} T_{\sigma}(\epsilon, V)\left(-\frac{\partial f_{\mathrm{STM}}(\epsilon, V)}{\partial V}\right) d \epsilon
$$

where $f_{\mathrm{STM}}(\epsilon)$ is the tip Fermi distribution function, $T_{\sigma}(\epsilon, V)=\operatorname{Tr}\left[\Gamma_{\sigma}^{\mathrm{C}}(\epsilon) G_{\sigma}(\epsilon, V) \Gamma_{\sigma}^{\mathrm{STM}}(\epsilon+e V) G_{\sigma}^{\dagger}(\epsilon, V)\right]$ is the Landauer transmission function that can be calculated from the device Green's function, Eq. (9), and the broadening functions which can be expressed for electrode $\alpha(=\mathrm{C}$ or STM $)$ as $\Gamma_{\sigma}^{\alpha}=-2 \operatorname{Im}\left(\Sigma_{\sigma}^{\alpha}\right)$. In Eq. 10, the voltage dependence of transmission function has been ignored, because the transmission values are not sensitive to low value bias voltages.

\section{B. Results}

The differential conductance is the most direct way to measure the energy-dependent LDOS of a system. In the 
present system, the calculated differential conductance, $\mathcal{G}(V)=\mathcal{G}_{\uparrow}(V)+\mathcal{G}_{\downarrow}(V)$ vs. sample bias $V$ for different applied gate voltages is shown in Fig. 4. Note that, the sample bias, $V$, means the voltage of the graphene with respect to the tip. In the present study, the graphene remains at the zero potential and the tip is at negative voltages, similarly to the experiment. Hence, when a negative voltage is applied to the tip, the sample bias is positive $(V>0)$ [43]. Furthermore, the gate-dependent $\mathcal{G}(V)$ spectra in the experiment, were measured at negative gate voltages. The measurements showed that, when the gate voltage moves to more negative values, the Dirac point position shifts to positive energies [see Fig. $2 \mathrm{~b}$ in Ref. [43]]. This behavior is clearly equivalent to application of positive $E_{G}$ values as we have done in Section IV and can be seen in Fig. 3 .

The calculated differential conductance $\mathcal{G}(V)$ shows a sharp peak at $E_{G}=0$ that comes from the Co spindown resonant state. The height of this peak decreases while the spectrum is broadened as the gate voltage is varied and $\left|E_{G}\right|$ increases. This effect is due to the nature of resonant states induced in the graphene near the Dirac point by the presence of an adsorbate; as can be seen in Fig. 3 these resonant states become narrower and stronger as the associated energy level of the Co adsorbate approaches the Dirac point energy, i.e., $E_{G} \rightarrow 0$. When $\left|E_{G}\right|$ increases, the cobalt orbital goes out of resonance with the graphene density of states peak in the vicinity of the Dirac point and the sharp conductance peak is suppressed.

In the experiment of Brar et al. [43], it has been demonstrated that the difference between the location of Dirac point voltage (marked $\mathrm{V}_{\mathrm{D}}$ in Fig. 2b of Ref. [43]), associated with the Dirac point energy [65], and the peak in the differential conductance signal (marked $\mathrm{S}$ in Fig. 2a and 2b of Ref. 43) increases as the magnitude of experimental gate voltage is increased. This enhancement in the difference between the Dirac point energy and the energy of the S state with increasing the gate voltage is in good agreement with the result of Fig. 3 which shows that, although with increasing $E_{G}$ both the cobalt-induced resonance feature (blue arrows) and the Dirac point energy (red arrow) in the LDOS spectrum of Fig. 3 move in the same direction, the Dirac point location moves more rapidly and the difference in energy between both locations (red and blue arrows) increases with $E_{G}$.

The experimental data [43] exhibits a sharp resonant peak $\mathrm{S}$ in the $\mathcal{G}(V)$ spectrum at low gate voltages in Fig. 2a of Ref. 43. With increasing absolute value of the gate voltage, the height of the peak decreases and the peak moves to lower sample bias. The sharp peak seen for $E_{G}=0$ in our model (Fig.4), is similar to the observed resonant peak $\mathrm{S}$ in Fig. 2a of Ref. 43 at low gate voltages and evolves with gate voltage in a qualitatively similar way as well.

The peaks in the $\mathcal{G}(V)$ spectra in Fig.4 occur at the sample bias voltages at which the STM tip Fermi en- ergy crosses the peaks of the partial densities of states that are due to the Co d $E_{1}$ state and the associated Coinduced graphene resonant state that are seen in Fig.3 a2-d2. These density of states features in Fig.3 a2-d2 and the peaks in $\mathcal{G}(V)$ spectra in Fig.4 are strongest and narrowest when the energy of the Co d $E_{1}$ state coincides with the graphene Dirac point energy, which occurs for $E_{G}=0$. The same is true of the Dirac point resonance peak of the $T$-matrix as can be seen in Fig. 2 and its inset. The large increase in the intensity of the experimentally observed resonant peak S in Fig. 2a of Ref. 43 (with decreasing absolute value of the gate voltage) also occurs as the S-peak energy approaches that of the graphene Dirac point energy (labelled $V_{D}$ ) in Fig. 2b of Ref. 43. We conclude that the large increase in the intensity of the resonant peak S in Fig. 2a of Ref. 43 with decreasing absolute value of the gate voltage is the experimental STM signature of the strengthening and narrowing of Dirac point resonance induced in the graphene by the Co (and of the associated Co level as well) as the Co level crosses the graphene Dirac point energy. Thus we propose that STM is able to detect the presence of adsorbate-induced Dirac point resonances in the graphene by monitoring the intensity of conductance peaks associated with orbitals of the adsorbate as those adsorbate levels cross the graphene Dirac point with changing gate voltage. Systematic experimental studies of adsorbate-induced Dirac point resonances in graphene based on this finding would be of considerable interest since experimental studies of these resonances have, to our knowledge, not been reported previously despite the considerable theoretical interest[7-29] in this topic.

The gate dependence of $\mathcal{G}(V)$ also indicates that the value of $E_{G}$ determines the ionization state of the Co atom, because by changing the value of $E_{G}$, the Co $3 d$ states can cross the Fermi energy and accordingly the charge occupation in these states changes. Therefore, the application of gate voltage enables tuning the chargecarrier density of graphene and hence the variation of Co adatom ionization state.

In the present model, the distance between the tip and the Co atom is $h=4 \AA$. Therefore, the overlap and hence the direct coupling between the Co atom and the tip is weak and that between the tip and the graphene is much weaker still. The reason is that, the sum of $\mathrm{Pt}$ and Co atomic radii is $\sim 2.64 \AA$ which is considerably less than $h$ which results in a small overlap between their atomic wave functions. In reality, the Co levels couple the STM tip to the graphene to open a conductive channel for electron transport. Since the coupling between the $E_{1}$ levels of adsorbate and the STM tip is weak the transport process for such levels is tunneling and the differential conductance spectra are quite similar to the $E_{1}$ resonance state LDOS seen in Fig. 3. From the extended Hückel theory hopping parameters between the Co atom and the Pt atom, we found that the $s$-levels of both atoms hybridize more strongly than that the other levels. The hopping energy between $s$-levels is roughly one order of 


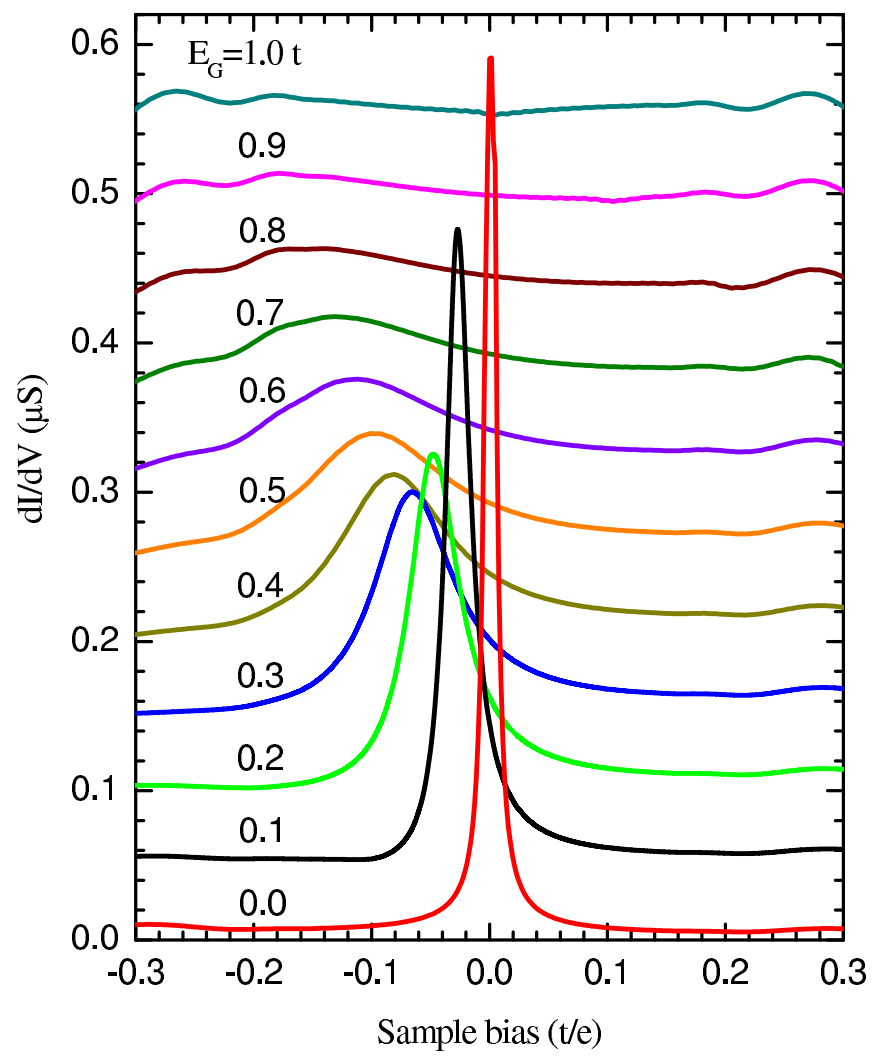

FIG. 4. (Color online) Calculated total differential conductance vs. sample bias for the graphene-cobalt-STM system, shown in Fig.1, at various $E_{G}$ values. The curves are shifted by offsets of $0.05 \mu S$ with respect to each other.

magnitude greater than the hopping energy between $E_{1^{-}}$ levels. Note that, in this work because of the position of the Co $E_{1}$-level relative to the graphene Dirac point and also to the other levels such as Co $4 s$-level, the $E_{1}$ level plays the main role in the tunneling transport through the cobalt atom at low values of the gate voltage.

Finally, it should be mentioned that we also examined the electron transmission through the Co adatom in the graphene-Co-Pt structure using the Landauer theory and the Lippmann-Schwinger equation [66] in which the EMOs included in the graphene were linear combinations of the atomic valence orbitals of the Co atom and the $2 s, 2 p_{x}$, and $2 p_{y}$ valence orbitals of graphene carbon atoms. The results, except with a small shift in the energy of the resonant states, were the same that we obtained based on the present method. This confirms that by the inclusion of just carbon $2 p_{z}$ orbitals in the graphene self-energy, we can obtain the same resonance feature in the conductance spectrum that we obtained based on the Lippmann-Schwinger approach.

\section{DISCUSSION}

In this study, we have presented a tight-binding theory of the Dirac point resonances due to a single Co adatom on gated graphene based on the $\pi$-band tight-binding description of the graphene plane with nearest neighbor hopping energy $t$ and the extended Hückel model of the adsorbate and the adsorbate-induced local $s p^{3}$ rehybridization of the graphene. Using the generalized effective Hamiltonian [29] for the relaxed geometry of Cographene system based on ab initio density functional theory, we found a strong spin-dependent scattering resonance at the Dirac point of graphene and a gate dependence of this resonance. This resonance arises from a hybridization of Co atomic orbitals with graphene states and such resonances have been predicted for $3 d$ transition metals adsorbed on graphene $[7,9,11-13]$. The energy position of the Co atomic levels with respect to the graphene Fermi energy determines the ionization state of the adsorbate. Because of spin-splitting of Co $4 s$ and $3 d$ orbitals, this ionization is different for spin-up and spin-down states. The results showed that, the Co $E_{1}$ state can be emptied with electrons as the state shifts above the Fermi energy through application of gate voltage, in agreement with the recent experiment [43]. The change in the occupation of the Co $3 d$ orbitals and hence in the ionization state of the adatom by application of gate voltage has also been predicted using DFT-based calculations for periodic systems. [12, 13].

Furthermore, we presented a formalism that we used to model the differential conductance measurements. Our calculation shows that, when the Co adatom states cross the Dirac point, a sharp feature is induced in the $\mathcal{G}(V)$ spectrum which is consistent with experiment and has not been addressed theoretically, previously. The height, broadening and the position of this feature is strongly dependent on gate voltage. The agreement between this theoretical dependence and the experimentally observed behavior of the S-peak in the STM differential conductance spectrum [43] allows us to attribute the S-peak to tunneling between the STM tip and a cobalt-induced Dirac point resonant state of the graphene, via a Co $3 d$ orbital. Systematic experimental studies of adsorbateinduced Dirac point resonances in graphene based on this finding would be of interest.

Our results also show the Dirac point energy of graphene and the cobalt-induced resonance feature in the differential conductance spectrum to move in different ways when a gate voltage is applied. Therefore the ionization state of Co adatom can be tuned by application of a gate voltage which induces a change in the occupation of the Co $3 d$ states. From the above findings regarding the $T$-matrix and the electronic density of states we can conclude that the Co adatom on graphene has a strongly spin-polarized LDOS especially at electron energies in the vicinity of the Dirac point of graphene. This suggests that Co adatoms on gated graphene may have possible applications in magnetic data storage and magnetic sen- 
sors and other applications which may be discovered.

\section{ACKNOWLEDGEMENTS}

We thank M. Crommie for helpful correspondence. This work was supported by NSERC, CIFAR, WestGrid, and Compute Canada.
[1] K.S. Novoselov, A.K. Geim, S.V. Morozov, D. Jiang, M.I. Katsnelson, I.V. Grigorieva, S.V. Dubonos, and A. A. Firsov, Nature (London) 438, 197 (2005).

[2] Y. Zhang, Y.-W. Tan, H.L. Stormer, and P. Kim, Nature (London) 438, 201 (2005).

[3] A. K. Geim and K. S. Novoselov, Nat. Mater. 6, 183 (2007).

[4] A. K. Geim, Science 324, 1530 (2009).

[5] P. R. Wallace, Phys. Rev. 71, 622 (1947).

[6] A. H. Castro Neto, F. Guinea, N. M. R. Peres, K. S. Novoselov, and A. K. Geim, Rev. Mod. Phys. 81, 109 (2009).

[7] Y. Mao, J. Yuan, and J. Zhong, J. Phys: Condens. Matter 20, 115209 (2008).

[8] K. T. Chan, J. B. Neaton, and M. L. Cohen, Phys. Rev. B 77, 235430 (2008).

[9] T.O. Wehling, A.V. Balatsky, M.I. Katsnelson, A.I. Lichtenstein, and A. Rosch, Phys. Rev. B 81, 115427 (2010).

[10] T. O. Wehling, H. P. Dahal, A. I. Lichtenstein, M. I. Katsnelson, H. C. Manoharan, and A. V. Balatsky, Phys. Rev. B 81, 085413 (2010).

[11] C. Cao, M. Wu, J. Jiang, and H.-P. Cheng, Phys. Rev. B 81, 205424 (2010).

[12] D. Jacob and G. Kotliar, Phys. Rev. B 82, 085423 (2010).

[13] K.T. Chan, H. Lee, and M.L. Cohen, Phys. Rev. B 83, 035405 (2011).

[14] T. G. Rappoport, M. Godoy, B. Uchoa, R. R. dos Santos and A. H. Castro Neto, Eurphys. Lett. 96, 27010 (2011).

[15] T. O. Wehling, A. I. Lichtenstein, M. I. Katsnelson, Phys. Rev. B 84, 235110 (2011).

[16] X. Liu, C. Z. Wang, Y. X. Yao, W. C. Lu, M. Hupalo, M. C. Tringides, and K. M. Ho, Phys. Rev. B 83, 235411 (2011).

[17] Yu.V. Skrypnyk and V.M. Loktev,Phys. Rev. B 73, 241402 (2006).

[18] V. M. Pereira, F. Guinea, J. M. B. Lopes dos Santos, N. M. R. Peres, and A. H. Castro Neto, Phys. Rev. Lett. 96, 036801 (2006).

[19] Yu. V. Skrypnyk and V.M. Loktev, Low Temp. Phys. 33, 762 (2007).

[20] T. O. Wehling, A. V. Balatsky, M. I. Katsnelson, A. I. Lichtenstein, K. Scharnberg, and R. Wiesendanger, Phys. Rev. B 75, 125425 (2007).

[21] J.P. Robinson, H. Schomerus, L. Oroszlány, and V.I. Fal'ko, Phys. Rev. Lett. 101, 196803 (2008).

[22] V. M. Pereira, J. M. B. Lopes dos Santos, and A. H. Castro Neto, Phys. Rev. B 77, 115109 (2008).

[23] D. M. Basko, Phys. Rev. B 78, 115432 (2008).

[24] T. O. Wehling, M. I. Katsnelson, and A. I. Lichtenstein, Chem. Phys. Lett. 476, 125 (2009).

[25] T. O.Wehling, M. I. Katsnelson, and A. I. Lichtenstein,
Phys. Rev. B 80, 085428 (2009).

[26] S. S. Pershoguba, Yu. V. Skrypnyk, and V. M. Loktev, Phys. Rev. B 80, 214201 (2009).

[27] T. O. Wehling, S. Yuan, A. I. Lichtenstein, A. K. Geim, and M. I. Katsnelson, Phys. Rev. Lett. 105, 056802 (2010).

[28] Yu. V. Skrypnyk and V. M. Loktev, Phys. Rev. B 82, 085436 (2010).

[29] S. Ihnatsenka and G. Kirczenow, Phys. Rev. B 83, 245442 (2011).

[30] F. Schedin, A. K. Geim, S. V. Morozov, E. W. Hill, P. Blake, M. I. Katsnelson, and K. S. Novoselov, Nature Mater. 6, 652 (2007).

[31] T. O. Wehling, K. S. Novoselov, S. V. Morozov, E. E. Vdovin, M. I. Katsnelson, A. K. Geim, and A. I. Lichtenstein, Nano Lett. 8, 173 (2008).

[32] O. Leenaerts, B. Partoens and F. M. Peeters, Phys. Rev. B 77, 125416 (2008); Appl. Phys. Lett. 92, 243125 (2008); Phys. Rev. B 79, 235440 (2009).

[33] H. E. Romero, P. Joshi, A. K. Gupta, H. R. Gutierrez, M. W. Cole, S. A. Tadigadapa, and P. C. Eklund, Nanotechnology 20, 245501 (2009).

[34] Y. H. Zhang, Y. B. Chen, K. G. Zhou, C. H. Liu, J. Zeng, H. L. Zhang, and Y. Peng, Nanotechnology 20, 185504 (2009).

[35] Z.M. Ao, F.M. Peeters, J. Phys. Chem. C114, 14503 (2010); Phys. Rev. B 81, 205406 (2010).

[36] B. Song, G. Cuniberti, S. Sanvito, and H. Fang, Appl. Phys. Lett. 100, 063101 (2012).

[37] K. S. Novoselov, A. K. Geim, S. V. Morozov, D. Jiang, Y. Zhang, S. V. Dubonos, I. V. Grigorieva, and A. A. Firsov, Science 306, 666 (2004).

[38] A. Das, S. Pisana, , B. Chakraborty, S. Piscanec, S. K. Saha, U. V. Waghmare, K. S. Novoselov, H. R. Krishnamurthy, A. K. Geim, A. C. Ferrari, and A. K. Sood, Nat. Nanotechnol. 3, 210 (2008).

[39] J. B. Oostinga, H. B. Heersche, X. L. Liu, A. F. Morpurgo, L. M. K. Vandersypen, Nat. Mater. 7, 151 (2008).

[40] Y. B. Zhang, T. T. Tang, C. Girit, Z. Hao, M. C. Martin, A. Zettl, M. F. Crommie, Y. R. Shen, F. Wang, Nature 459, 820 (2009).

[41] R. Xiao, D. Fritsch, M. D. Kuz'min, K. Koepernik, H. Eschrig, M. Richter, K. Vietze, and G. Seifert, Phys. Rev. Lett. 103, 187201 (2009).

[42] M. Sargolzaei and F. Gudarzi, J. Appl. Phys. 110, 064303 (2011).

[43] V.W. Brar, R. Decker, H. Solowan, Y. Wang, L. Maserati, K.T. Chan, H. Lee, C.O. Girit, A. Zettl, S.G. Louie, M.L. Cohen, and M.F. Crommie, Nat. Phys. 7, 43 (2011).

[44] B. C. Stipe, M. A. Rezaei, W. Ho, Science 280, 1732 (1998). 
[45] X. H. Qiu, G. V. Nazin, and W. Ho, Phys. Rev. Lett. 92, 206102 (2004).

[46] T. O. Wehling, I. Grigorenko, A. I. Lichtenstein, A. V. Balatsky, Phys. Rev. Lett. 101, 216803 (2008).

[47] F. Demir and G. Kirczenow, J. Chem. Phys. 134, 121103 (2011).

[48] D. Eom, D. Prezzi, K. T. Rim, H. Zhou, M. Lefenfeld, S. Xiao, C. Nuckolls, M. S. Hybertsen, T. F. Heinz, and G. W. Flynn, Nano Lett. 9, 2844 (2009).

[49] M. Vanin, J. J. Mortensen, A. K. Kelkkanen, J. M. Garcia-Lastra, K. S. Thygesen, and K. W. Jacobsen, 81, 081408(R) (2010).

[50] W. X. Wang, S. H. Liang, T. Yu, D. H. Li, Y. B. Li, and X. F. Han, 109, 07C501 (2011).

[51] For a recent review, see G. Kirczenow, in The Oxford Handbook of Nanoscience and Technology, Volume I: Basic Aspects, edited by A. V. Narlikar and Y. Y. Fu (Oxford University Press, Oxford, UK, 2010), Chap. 4.

[52] J. H. Ammeter, H. B. Buergi, J. C. Thibeault, and R. Hoffmann, J. Am. Chem. Soc. 100, 3686 (1978).

[53] The YAEHMOP code implementation of the extended Huckel theory (Ref. [52]) by G. A. Landrum and W. V. Glassey (Source-Forge, Fremont, California, 2001) was used. YAEHMOP does not include spin effects.

[54] H. Raza and E. C. Kan, Phys. Rev. B 77, 245434 (2008); J. Comput. Electron. 7, 372 (2008).

[55] G. Kirczenow and S. Ihnatsenka in Graphene Nanoelectronics: Metrology, Synthesis, Properties and Applica- tions edited by H. Raza (Springer, Berlin Heidelberg New York, 2012).

[56] D. Kienle, J. I. Cerda, and A. W. Ghosh, J. Appl. Phys. 100, 043714 (2006).

[57] E. Emberly and G. Kirczenow, Phys. Rev. Lett. 81, 5205 (1998).

[58] E. Emberly and G. Kirczenow, J. Phys. Condens. Matter 11, 6911 (1999).

[59] For a discussion of the relevant physics see E. G. Emberly, G. Kirczenow, Chem. Phys. 281, 311 (2002), Appendix A

[60] J. O. Sofo, A. M. Suarez, G. Usaj, P. S. Cornaglia, A. D. Hernández-Nieves, and C. A. Balseiro, Phys. Rev. B 83, 081411(R) (2011).

[61] J. O. Sofo, G. Usaj, P. S. Cornaglia, A. M. Suarez, A. D. Hernández-Nieves, and C. A. Balseiro, Phys. Rev. B 85, 115405 (2012).

[62] G. S. Painter and D.E. Ellis, Phys. Rev. B 1, 4747 (1970).

[63] S. Datta, Quantum Transport: Atom to Transistor (Cambridge University Press, Camberige, 2005).

[64] A. Saffarzadeh, J. Appl. Phys. 103, 083705 (2008); 104, 123715 (2008).

[65] Y. Zhang, V. W. Brar, F. Wang, C. Girit, Y. Yayon, M. Panlasigui, A. Zettl, and M. F. Crommie, Nat. Phys. 4, 627 (2008).

[66] H. Dalgleish and G. Kirczenow, Phys. Rev. B 72, 155429 (2005). 